\title{
Follow-up of patients with uncontrolled asthma: clinical features of asthma patients according to the level of control achieved (the COAS study)
}

\author{
Rosa Munoz-Cano (1) ${ }^{1}$, Alfons Torrego², Joan Bartra', Jaime Sanchez-Lopez', \\ Rosa Palomino ${ }^{3}$, Cesar Picado ${ }^{1}$ and Antonio Valero ${ }^{1}$
}

Affiliations: ${ }^{1}$ Dept of Pulmonology and Respiratory Allergy, Hospital Clinic Barcelona, Institut d'Investigacions Biomèdiques August Pi i Sunyer (IDIBAPS), Centro de Investigaciones Biomedicas en Red de Enfermedades Respiratorias (CIBERES), Universitat de Barcelona, Barcelona, Spain. ${ }^{2}$ Dept of Pulmonology, Hospital de la Santa Creu i San Pau, Barcelona, Spain. ${ }^{3}$ Gestió Organització Comunicació S.A. (GOC), Barcelona, Spain.

Correspondence: Rosa Munoz-Cano, Dept of Pulmonology and Respiratory Allergy, Hospital Clinic, C/Villarroel 170, Barcelona 080036, Spain. E-mail: rmunozaclinic.ub.es

@ERSpublications

Asthma in most patients is not fully controlled despite adjusting their treatment using GINA 2010 strategy criteria http://ow.ly/C73c307fMOZ

Cite this article as: Munoz-Cano R, Torrego A, Bartra J, et al. Follow-up of patients with uncontrolled asthma: clinical features of asthma patients according to the level of control achieved (the COAS study). Eur Respir J 2017; 49: 1501885 [https://doi.org/10.1183/13993003.01885-2015].

ABSTRACT Our aim was to study the asthma control achieved in patients with uncontrolled asthma who had received appropriate treatment according to the Global Initiative for Asthma (GINA) 2010 (valid at the time the study was designed), and to analyse the factors associated with a lack of asthma control.

This was a multicentre study in routine clinical practice performed in patients with uncontrolled asthma according to GINA 2010. At visit 1, we recorded demographics, asthma characteristics and spirometry. We assessed asthma control using GINA 2010 criteria and the Asthma Control Test (ACT). Treatment was optimised according to GINA 2010. At visit 2, 3 months later, we reassessed spirometry, asthma control and factors associated with failure to achieve control.

We recruited 1299 patients with uncontrolled asthma (mean age $46.5 \pm 17.3$ years, $60.7 \%$ women, $25.8 \%$ obese). The mean percentage of predicted forced expiratory volume in $1 \mathrm{~s}$ was $76.4 \pm 12.8 \%$ and the mean post-bronchodilator increase was $14.9 \pm 6.8 \%$. We observed poor agreement between ACT and GINA 2010 when evaluating asthma control (kappa $=-0.151$ ). At visit 2, asthma in $71.2 \%$ of patients was still not fully controlled. Patients whose asthma remained uncontrolled were older, had a higher body mass index, greater disease severity, longer disease evolution and worse lung function.

After treatment optimisation, most patients did not achieve optimal control according to GINA 2010. Risk factors for failure to achieve asthma control were time of disease evolution, severity, age, weight and lung function impairment (excluded in the GINA 2014).

Received: Nov 122015 | Accepted after revision: Nov 282016

Conflict of interest: Disclosures can be found alongside this article at erj.ersjournals.com

Support statement: This work was financially supported by laboratories Chiesi. Funding information for this article has been deposited with the Open Funder Registry.

Copyright OERS 2017 


\section{Introduction}

The GINA (Global Initiative for Asthma) strategy [1], revised in 2010 and more recently in 2014 [2], estimated that over 300 million people worldwide have asthma. Despite the availability of effective medication, the disease remains uncontrolled in many patients and has a large impact on their health-related quality of life. The CHAS study [3], conducted in a population visiting primary care centres, found a prevalence of uncontrolled asthma of 63.9\% (95\% CI 61.9-65.9\%). Another study conducted in Europe (France, Germany, Italy, Spain and UK) in 2006, 2008 and 2010 found controlled asthma in 55\%, $56.6 \%$ and $53.5 \%$ of patients, respectively [4]. A recent study, Measuring Asthma GINA Control Study (MAGIC) [5], conducted in Spain with 1363 asthma patients in primary care, pulmonology and allergy clinics, found that only $13.6 \%$ had well-controlled asthma according to GINA criteria.

Several authors have described the existence of factors associated with poor asthma control. The effects of age, sex, educational level and smoking are controversial because some studies have shown a positive association between these factors and a lack of control whereas others have not [6-10]. Interestingly, one previous study showed that disease perception affects asthma control [11]. Frequently, patients overestimate their level of control, which can result in poor treatment adherence [7].

Given the importance of asthma control, it is essential to establish the efficacy of prescribed therapeutic interventions. One of the assessment tools available is the Asthma Control Test (ACT) [12-15], an easy-to-apply questionnaire for patients older than 12 years.

The aim of the present study, named the COAS study, was to assess the level of control achieved in patients with uncontrolled asthma after receiving appropriate treatment for 3 months according to the GINA 2010 strategy [1]. The study also evaluated the factors associated with a lack of asthma control.

\section{Methods}

\section{Study design}

This cross-sectional multicentre study was conducted in routine clinical practice in specialised allergy and pulmonology centres in Spain. Functional, clinical and epidemiological variables were obtained at baseline (visit 1) and 3 months after treatment optimisation (visit 2).

\section{Study population}

Patients of both sexes between 18 and 75 years old with inadequately controlled asthma (partially and not controlled), defined according to the GINA 2010 strategy, were recruited. The exclusion criteria were cardiopathy, untreated gastro-oesophageal reflux or respiratory disease other than asthma, and treatment with oral corticosteroids or anti-IgE. Patients with chronic obstructive pulmonary disease were excluded on the basis of a clinical history of persistent cough and age $>40$ years in the case of active or ex-smokers, in accordance with the Global Initiative for Chronic Obstructive Lung Disease (GOLD) criteria [16].

To calculate the number of patients required for the study, the corresponding formula was used to estimate a percentage in infinite samples. Establishing an accuracy level of $2.5 \%$ and a confidence level of $95 \%$, the resulting minimum size was 1534 . Assuming a percentage loss of $15 \%$, the total number of patients needed was 1750 .

\section{Research centres}

A total of 317 investigators from all over Spain participated in the study. Each of the investigators involved was required to include five patients.

\section{Endpoints of the study}

The recruitment period lasted 3 months. Patients were included consecutively after attending visits at their health centre.

At visit 1, patients were assessed following standard clinical practice. The patient's medical history and demographic and anthropometric data were collected, including asthma onset, treatment received in the last 3 months, their current treatment step according to GINA 2010 and their new treatment step after treatment adjustment.

Comorbidities such as rhinitis (allergic sensitisation, severity and duration), sinonasal polyposis, nonsteroidal anti-inflammatory drug (NSAID) hypersensitivity and smoking habit were assessed, and patients' knowledge of their disease and adherence to asthma treatment were evaluated via seven questions.

Spirometry and a bronchodilator test were also performed. Asthma control was assessed using GINA 2010 criteria and the Spanish version of the validated ACT questionnaire [1]. The GINA 2010 asthma control questionnaire consists of four questions which assess daytime symptoms, night waking due to asthma, reliever 
medication needed and any activity limitation due to asthma in the past 4 weeks. The questions are scored yes or no. Four affirmative answers define well-controlled asthma, one or two affirmative answers defines partially controlled asthma, and three to four negative answers defines uncontrolled asthma [1]. The ACT questionnaire consists of five questions assessing limitations on activity, dyspnoea, nocturnal symptoms, use of rescue medication and overall rating of patients' asthma control during the previous 4 weeks. The questions are scored from 1 (worst) to 5 (best) and the total score is the sum of the responses, which ranges from 5 (poor control) to 25 (total control). ACT scores $\geqslant 20$ define well-controlled asthma during the previous 4 weeks, scores of 16-19 define partially controlled asthma, and scores $\leqslant 15$ define uncontrolled asthma.

At visit 2, 3 months after treatment optimisation, the medical history was re-evaluated, a new spirometry and bronchodilator test were performed, and asthma control was reassessed using GINA criteria and ACT. Patients' knowledge of their disease and their adherence to asthma treatment was re-evaluated. Treatment was again modified in patients with partially controlled and not controlled asthma.

\section{Legal provisions and ethics}

Investigators were instructed in the ethical principles and agreement of the Helsinki Declaration and briefed in accordance with international regulations on conducting epidemiological studies, contained in the "International Guidelines for Ethical Review of Epidemiological Studies" [17] and the recommendations of the Spanish Society of Epidemiology. The processing of patients' data complied with the Spanish Protection of Personal Data Act of 1999. The study was approved by the Ethics Committee of the Hospital Clinic of Barcelona and all patients gave informed consent prior to enrolment.

\section{Statistical analysis}

Data were entered by the investigators themselves on case report forms at each centre. The study was monitored for compliance with its basic endpoints. The database, produced from the aggregate record of the case reports forms, was managed using Access (Microsoft Co., Redmond, WA, USA), applying the appropriate filters and validation rules to ensure maximum data reliability. For the descriptive treatment of the qualitative variables of the study, the absolute (n) and relative frequencies were used as percentage (\%). For the analysis of quantitative variables, the normality of their distribution was checked, using the Kolmogorov-Smirnov test; if the data were normally distributed, the mean was used as a measure of central tendency and standard deviation as a measure of dispersion; for data that were not normally distributed, the median was used for central tendency and the interquartile range as a measure of dispersion. To establish the association between qualitative variables with the main control variable, contingency tables were developed using the Chi-squared test if the sample size was large enough and otherwise using Fisher's exact test. To examine mean differences in the control groups, a t-test or one-way ANOVA was used. If the variables were not normally distributed, the non-parametric Mann-Whitney or Kruskal-Wallis test was performed. The kappa index was used to calculate agreement. In all cases the level of statistical significance was $5 \%(\alpha=0.05)$. The analysis was performed with SPSS (Statistical Package For Social Sciences) version 17.0 (SPSS, Inc. Chicago, IL, USA).

\section{Results}

Study population

A total of 1412 patients were recruited by the 317 investigators but only 1299 (4.1 patients/investigator) were considered eligible. All patients had uncontrolled (partially and not controlled) asthma according to the GINA 2010 criteria.

Obesity, defined as a body mass index $(\mathrm{BMI})>30 \mathrm{~kg} \cdot \mathrm{m}^{-2}$, was present in $25.8 \%$ of patients $(\mathrm{n}=279)$. In addition, 52\% reported a respiratory allergy, the most prevalent allergens being mites (38\%), pollen (29\%), epithelia (15\%) and fungi (6\%) (table 1).

NSAID hypersensitivity was diagnosed in $10 \%$ of patients, in whom asthma was not controlled in $53.7 \%$, partially controlled in $23.9 \%$ and well-controlled in $22.4 \%$. Statistically significant differences $(\mathrm{p}=0.014)$ were found between groups. NSAIDs triggered asthma in $70 \%$ of patients with NSAID hypersensitivity, triggered both rhinitis and asthma in $27 \%$, and in rare cases triggered rhinitis alone (3\%). Eighty five patients had sinonasal polyposis (7\%) (table 1).

\section{Visit 1}

Asthma control

Although the patients selected had uncontrolled (partially and not controlled) asthma according to GINA 2010, 12.0\% were classified as having well-controlled asthma using ACT, with a mean ACT score of 21.0 \pm 1.3 . A total of $26.2 \%$ had partially controlled asthma (mean ACT score $17.4 \pm 1.2$ ) and in $61.8 \%$ the

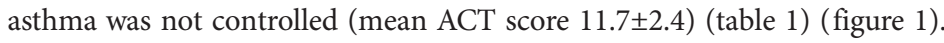


TABLE 1 Demographic, clinical and asthma control characteristics in subjects examined at baseline visit and second visit

\begin{tabular}{|c|c|c|c|c|c|}
\hline & \multirow{2}{*}{$\frac{\text { Visit } 1}{\text { Not controlled }}$} & \multicolumn{4}{|c|}{ Visit 2} \\
\hline & & Well controlled & Partially controlled & Not controlled & p-value \\
\hline Subjects $\mathrm{n}$ & 1299 & $426(32.8 \%)$ & $308(27.7 \%)$ & $565(43.5 \%)$ & - \\
\hline Women & 60.7 & 63.8 & 60.3 & 58.7 & NS \\
\hline Body mass index $\mathrm{kg} \cdot \mathrm{m}^{-2}$ & $26.9 \pm 5.5$ & $26.2 \pm 5.1$ & $27.0 \pm 5.7$ & $27.4 \pm 5.6$ & $0.007^{\#}$ \\
\hline \multicolumn{6}{|l|}{ Smoking status } \\
\hline Smokers & 13.8 & 13.8 & 13.0 & 14.2 & \\
\hline Diagnosis of respiratory allergy & 52.0 & 54.2 & 50.0 & 51.3 & NS \\
\hline Rhinitis & 51.3 & 52.6 & 53.6 & 49.2 & NS \\
\hline Years of evolution & $13.3 \pm 9.9$ & $12.2 \pm 9.2$ & $13.6 \pm 10.2$ & $14.1 \pm 10.1$ & NS \\
\hline \multicolumn{6}{|l|}{ Severity of rhinitis } \\
\hline Mild & 15.7 & 20.1 & 10.9 & 15.1 & NS \\
\hline Persistent & 52.5 & 50.7 & 56.8 & 51.5 & \\
\hline Asthma years of evolution & $14.2 \pm 12.2$ & $12.5 \pm 10.4$ & $14.9 \pm 11.3$ & $15.1 \pm 13.6$ & $0.005^{\#, \uparrow_{1}+}$ \\
\hline \multicolumn{6}{|l|}{ Asthma classification (GINA) } \\
\hline Mild intermittent & 0.4 & 0.3 & 0.0 & 0.8 & $<0.001$ \\
\hline Mild persistent & 6.4 & 7.2 & 4.1 & 7.1 & \\
\hline Moderate persistent & 82.1 & 88.2 & 85.9 & 75.5 & \\
\hline Severe persistent & 11.0 & 4.4 & 10.0 & 16.7 & \\
\hline FVC \% predicted & $87.2 \pm 13.0$ & $93.0 \pm 18.5$ & $91.3 \pm 16.6$ & $85.4 \pm 19.7$ & $<0.001^{\#,+}$ \\
\hline FEV $1 \%$ predicted & $76.4 \pm 12.8$ & $87.9 \pm 20.8$ & $84.7 \pm 17.6$ & $77.3 \pm 20.2$ & $<0.001^{\#,+}$ \\
\hline Increase post-BD FEV $1 \%$ predicted & $14.9 \pm 6.8$ & $8.5 \pm 13.1$ & $10.2 \pm 13.6$ & $13.0 \pm 17.0$ & $<0.001^{\#}$ \\
\hline \multicolumn{6}{|l|}{ ACT } \\
\hline Controlled $(\geqslant 20)$ & 12.0 & 89.3 & 72.0 & 53.5 & $<0.001$ \\
\hline
\end{tabular}

Data are presented as \% or mean \pm SD, unless otherwise indicated. GINA: Global Initiative for Asthma; FVC: forced vital capacity; FEV1: forced

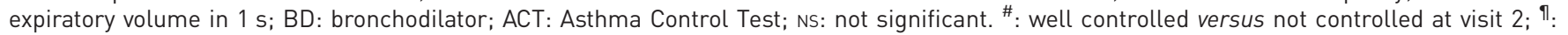
well controlled versus partially controlled at visit $2 ;^{+}$: partially controlled versus not controlled at visit 2.

According to the patients' opinions (obtained from the answer to the fifth question of the ACT questionnaire), $14.7 \%$ characterised their asthma as well controlled or completely controlled, $34 \%$ somewhat controlled, and 51.3\% not controlled or poorly controlled (table 2).

\section{Asthma treatment}

Table 3 shows the treatment received by patients at visit 1 and after optimising treatment (visit 2). At visit 1 , most patients were treated with inhaled corticosteroids (ICS) with a long-acting $\beta 2$-agonists (LABA) $(39.1 \%)$ or with ICS alone $(30.6 \%)$. Interestingly, even though all selected patients had uncontrolled asthma according to GINA 2010 criteria, only $66.7 \%$ were using short-acting $\beta 2$-agonists.

Figure 2 shows the treatment steps at visit 1 and visit 2 . At visit 1, 85.6\% of patients were advised to increase at least one step; in $14.4 \%$, the step was not changed because optimising the inhaler technique and treatment advice were considered sufficient to improve asthma control. A total of $59.2 \%$ of patients increased only one step, $20.4 \%$ increased two steps and 6\% increased more than two steps. At visit 1, most patients were at step 3 .

However, after optimising the treatment, most patients moved up to step 4. Thus, in most cases the treatment plan underestimated the patients' clinical status at visit 1 .

Knowledge of the disease and adherence to treatment

Table 4 shows the results of the seven-point questionnaire regarding the patients' knowledge of asthma and treatment. Seventy-eight per cent of patients stated they were familiar with their breathing 
FIGURE 1 Asthma control according to the Asthma Control Test (ACT) questionnaire at visit 1 and visit 2 . a) Statistically significant differences were found $(p<0.001)$ between visit 1 and 2 using McNemar's test. b) Individual ACT scores at visit 1. c) Individual ACT scores at visit 2 .
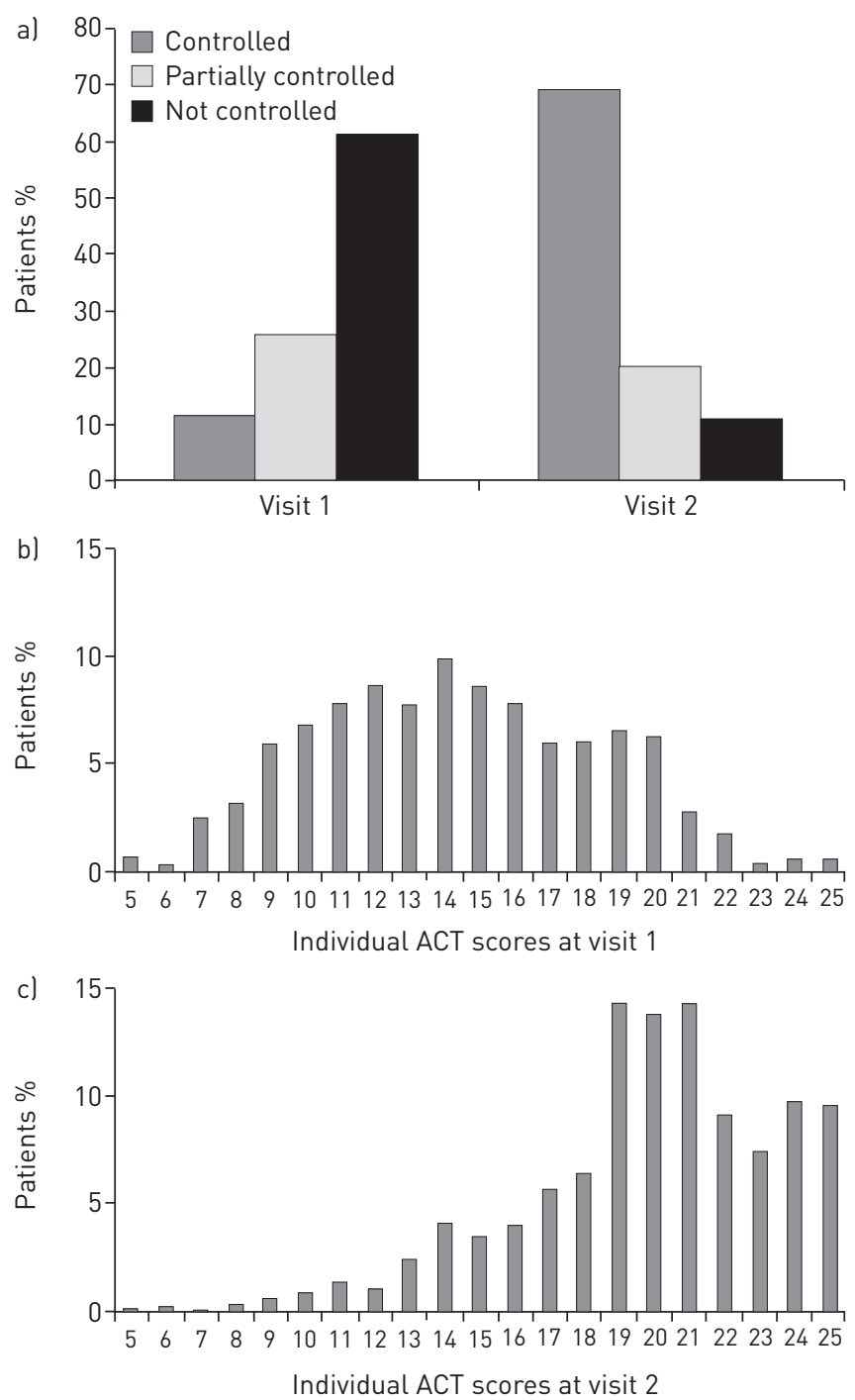

TABLE 2 Patient's opinion on asthma control at visit 1 and 2 according to the answer to question 5 of the Asthma Control Test questionnaire "How would you rate your asthma control during the past 4 weeks?"

Visit 1

Visit 2

p-value

Totally controlled

Well controlled

1.7

16.7

$<0.001$

Somewhat controlled

57.4

13.0

34.0

21.8

Poorly controlled

43.9

4.0

Not controlled

7.4

0.1

Data are presented as \%. ${ }^{\#}$ : p-value refers to comparison between visit 1 and 2 for each control level.

problem; $60 \%$ said that, based on experience, the inhaler made them breathe better; and $28 \%$ of the participants did not know the type of inhaler used. Most patients $(86 \%)$ had been taught by healthcare staff how to use the inhaler, but only $51 \%$ of patients used it correctly. Regarding knowledge of asthma treatment, 59\% had an ample or sufficient level of information and only 53\% had good adherence to treatment. 
TABLE 3 Treatment at visit 1 and visit 2 after therapeutic optimisation using Global Initiative for Asthma criteria

\begin{tabular}{lccc} 
& Treatment at visit 1 & Treatment at visit 2 & p-value $^{\#}$ \\
\hline Inhaled corticosteroids & 30.6 & 9.8 & $<0.001$ \\
Long-acting $\beta 2$ agonists & 11.5 & 6.0 & \\
$\beta 2$ agonists + corticosteroids & 39.1 & 83.9 & \\
Short-acting $\beta 2$ agonists & 66.7 & 48.4 & \\
Anti-leukotriene agents & 21.1 & 35.7 & \\
\hline
\end{tabular}

Data are presented as \%. ${ }^{*}$ : p-value refers to comparison between visit 1 and 2 for treatment.

FIGURE 2 Therapeutic step at visit 1 and visit 2 using the Global Initiative for Asthma strategy. Statistically significant differences were observed between groups using McNemar's test. \#: versus visit $1 ;$ १: versus visit 2 .

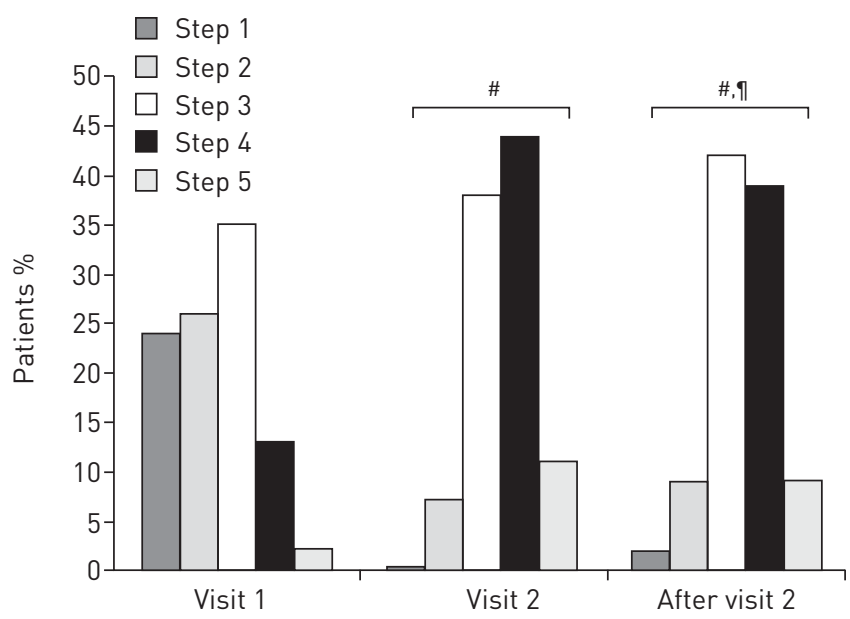

\section{Visit 2}

\section{Asthma control}

After 3 months of treatment (visit 2), 32.8\% of patients were classified as having well-controlled asthma, $27.7 \%$ had partially controlled asthma and $43.5 \%$ still had uncontrolled asthma according to GINA 2010 criteria (table 1). However, according to the ACT questionnaire, $69.2 \%$ had well-controlled asthma (mean

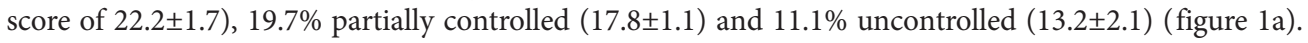

\section{TABLE 4 Patients' knowledge about their disease and asthma inhaled treatment at visit 1}

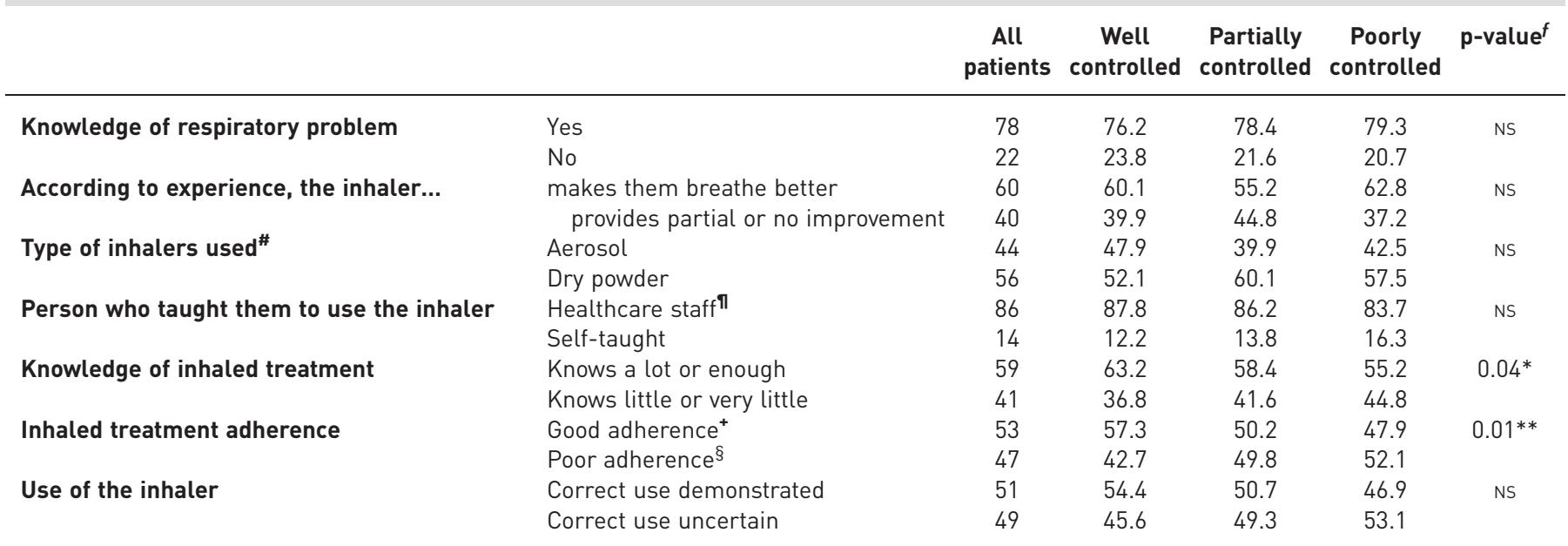

Data are presented as \%. NS: not significant. ${ }^{\#}: 28 \%$ did not know the type of inhaler used; ${ }^{\text {ๆ}: ~ d o c t o r, ~ n u r s e, ~ p h a r m a c i s t ; ~}{ }^{+}$: takes each day as directed; ${ }^{\S}$ : forgets to take it occasionally if feeling well, forgets or skips it often, or does not usually take it due to perceiving no benefit; $f_{\text {: }} \mathrm{p}$-value corresponds to the comparison between well controlled, partially controlled and not controlled at visit $1 .{ }^{*} p<0.05 ;{ }^{* *} p<0.01$. 
TABLE 5 Agreement in asthma control classification between Global Initiative for Asthma criteria versus Asthma Control Test questionnaire at visit 2 in all patients

\begin{tabular}{llccc} 
& & \multicolumn{3}{c}{ GINA criteria } \\
\cline { 3 - 5 } & & Well controlled & Partially controlled & Not controlled \\
\hline \multirow{2}{*}{ ACT } & Controlled & 28.1 & 17.2 & 23.9 \\
& Partially controlled & 2.8 & 5.4 & 11.5 \\
& Not controlled & 0.6 & 1.3 & 9.3 \\
\hline
\end{tabular}

Data are presented as \%. Degree of agreement k=-0.151. GINA: Global Initiative for Asthma; ACT: Asthma Control Test.

According to the GINA 2010 criteria, the increase in well-controlled asthma at visit 2 was only 32.8\%, whereas according to the ACT it was $57.2 \%$.

We found that only $28.1 \%$ of patients were classified as having well-controlled asthma by both the GINA 2010 criteria and the ACT questionnaire. The comparison between the two methods as assessed by the kappa index was low and negative $(\mathrm{k}=-0.151)$, indicating that there is no agreement between them (table 5).

According to the patients' opinions reported in the fifth question of the ACT questionnaire, most patients (74.1\%) believed that their asthma was well or totally controlled (table 2). Large discrepancies were found when comparing the classification obtained using the GINA 2010 criteria, the ACT and patients' perception: for instance, rates for "well controlled" ranged from 32.8\% using GINA through $69.2 \%$ using ACT to $74.1 \%$ considering only the patients' opinion. This suggests that the therapeutic strategy will differ substantially depending on the criteria used.

One of the reasons for poor control of asthma is poor adherence to treatment [18]. In our case, although at visit 2 treatment adherence had increased by more than $40 \%$ (the average rate in the three groups was $96.6 \%$ at visit 2 compared with $53.5 \%$ at visit $1 ; \mathrm{p}<0.001$ ), there were no differences between well-controlled, partially controlled and uncontrolled asthma (table 4).

\section{Asthma treatment}

After re-evaluation at the second visit, asthma in most patients (71.2\%) remained uncontrolled (partially and not controlled) (table 1). At visit 1, most patients were at step 3; after optimising treatment, most moved up to step 4 (figure 2).

Treatment optimisation mainly meant an increase in the use of fixed combinations of ICS+LABA, from a mean of $39.1 \%$ at visit 1 to $83.9 \%$ at visit 2. Anti-leukotriene use also increased (from $21.1 \%$ to $35.7 \%$ ) (table 3 ).

Because asthma remained uncontrolled in most patients, a new treatment optimisation was performed at visit 2. A one-step increase was required in $41.8 \%$ of patients and a two-step increase in $5.5 \%$ of patients (figure 2).

Regarding adherence to treatment, no differences were found between groups; poor adherence to treatment was shown in only $1.6 \%$ in the well-controlled group, $3.9 \%$ in the partially controlled group and $3.7 \%$ in the not controlled group.

\section{Knowledge of the disease}

Patients with lower treatment adherence $(\mathrm{p}<0.01)$ and poorer knowledge of the treatment $(\mathrm{p}<0.04)$ presented with worse asthma control at visit 2 . There was a tendency toward poorer control when patients were uncertain of the correct use of their inhaled treatment $(p<0.06)$. No other factors regarding asthma knowledge recorded at visit 1 were related to asthma control at visit 2 (table 4).

\section{Risks factors associated with asthma control}

More advanced age, higher BMI, early asthma onset, greater severity, worse spirometry values and higher percentage of bronchodilation were significantly associated with worse disease control. Sex, smoking, allergy diagnosis, duration, severity and duration of rhinitis were not associated with the degree of asthma control (table 1).

\section{Discussion}

We have evaluated the asthma control achieved after optimising treatment in a selected population of patients with uncontrolled asthma using the GINA 2010 criteria. Although treatment optimisation resulted 
in a significant increase in asthma control, asthma remained inadequately controlled in $71.2 \%$ of patients, partially controlled in $27.7 \%$ and not controlled in $43.5 \%$.

The GOAL study is the seminal work investigating the role of a stepwise strategy in asthma treatment and control [19]. Whereas GOAL was a 52-week clinical trial, our study was a 12-week real-life study, and these major methodological differences could account for the difference in the results. Whereas $51 \%$ of patients had well-controlled asthma at 12 weeks in GOAL, only $32.8 \%$ had well-controlled asthma in the present study.

Interestingly, in the GOAL study, the number of patients who achieved well-controlled asthma at 12 weeks was much higher than the number observed between 12 and 54 weeks, even though no further changes in treatment were introduced in this period [19]. This finding suggests that 12 weeks of stepwise increases in asthma treatment provides relevant information regarding the expected benefit of dose escalation based on international treatment strategies.

The percentage of patients with controlled asthma according to GINA criteria in the present study was similar to those obtained in other studies in Spain (29.9\% [20], 37.4\% [3] and 38.3\% [21]). However, a longitudinal study conducted in Europe (France, Germany, Italy, Spain and UK) in 2006, 2008 and 2010 using the ACT questionnaire found a higher percentage of patients with controlled asthm $(55 \%, 56.6 \%$ and $53.5 \%$, respectively) [21]. The results for the Spanish population did not exceed 50\%. In our study, $69.2 \%$ of our patients had controlled asthma at visit 2 according to the ACT questionnaire, indicating a $57 \%$ increase compared to visit 1 . Other studies using ACT have shown increases of $20 \%$, $23 \%$ and $26 \%$ in asthma control in patients followed at 1,3 and 6 months, respectively [22]. This indicates that close monitoring and treatment optimisation increase the percentage of subjects with controlled asthma. Geographical differences, characteristics of the asthmatic population selected and the criteria used to evaluate control (among other factors) could account for the differences observed between studies.

In our study, in most cases the treatment plan underestimated patients' clinical status at the first visit. In fact, most patients (85.6\%) needed an increase of at least one treatment step, which in most cases meant using fixed combinations of ICS+LABA. Other studies have also shown the relationship between better control and the increase of treatment steps, in particular with the use of ICS+LABA fixed combinations $[5,23,24]$. This suggests that under-treatment is a major cause of uncontrolled disease in asthma patients, along with lack of adherence [3].

Several variables have been identified to affect asthma control, including age, sex, ethnicity, body mass, smoking, educational level, physical activity, treatment with oral corticosteroids, disease severity, comorbidities, adherence, living in a rural environment and stress situations involving anxiety or depression. However, the effects of these factors are not consistent across studies [3, 4, 7-10, 22, 26-27]. In our study, older age, overweight/obesity, NSAID hypersensitivity, duration of disease, poorer lung function and greater bronchodilation were associated with poorer control, while sex, smoking, allergy diagnosis, and rhinitis prevalence and characteristics had no influence. Patients with uncontrolled asthma had worse lung function (forced expiratory volume in $1 \mathrm{~s}(\mathrm{FEV} 1)$ ), which could be the reason for the lack of control and could be associated with an asthma-chronic obstructive pulmonary disease overlap syndrome. However no differences were found between groups regarding smoking.

The GINA 2010 strategy [1], ACT and Asthma Control Questionnaire (ASQ) [28] are used to evaluate asthma control. Some studies have found the agreement between these methods to be low [29], and that both ACQ and ACT overestimate disease control when using GINA criteria as the "gold standard" [30]. The agreement found between GINA and ACT in a European multicentre study (Italy, Spain, USA, France, Germany and UK) showed an overall kappa index of 0.42 , with differences between the participating countries ranging between 0.29 and 0.52 [31]. In another study, the agreement between GINA criteria and ACQ-5 had a kappa index of 0.63 and ACQ-5 proved to be more sensitive to changes in clinical trials than GINA [32]. A potential explanation for the discordance found in our study between GINA and ACT is the fact that we used GINA 2010, which, unlike ACT, includes lung function. Actually, the between-visit variability in FEV1 limits its utility for adjusting treatment or evaluating symptom control. From 2014, GINA has recommended the use of spirometry primarily for identifying patients at increased risk of exacerbations, but not to evaluate symptom control [2]. Furthermore, the low agreement between physician-assessed asthma control and the GINA strategy has also been shown; a kappa index of 0.337 in some studies suggests that physicians overestimate both rates of asthma control and its severity [33]. Patients also overestimate control compared to doctors, with poor agreement between the two methods (kappa index of 0.53) [33]. For reasons that are unclear, our study found a very low correlation between the ACT and GINA criteria, with a kappa index of -0.15 , indicating a virtually null agreement between the two instruments. Considering their answers to question 5 of the ACT questionnaire, $48.7 \%$ of our patients reported that their asthma was well or completely controlled, but according to the GINA criteria not all these cases were controlled. In general, patients and physicians overestimate asthma control compared to the results of questionnaires (ACT, ACQ) 
and the GINA criteria; indeed, questionnaires also overestimate control when compared to the GINA criteria. The large differences observed between the methods highlights the importance of the choice of instrument when evaluating and treating patients or comparing studies. Many factors can contribute to the discrepancies observed, including the question of whether the tools were evaluated in similar populations and in similar settings as their intended use [34]. Given the limitations of the classic composite scores in the assessment of asthma control, several recent studies have proposed novel instruments, although the validity of these new tools remains to be demonstrated [35].

Many possible reasons exist for poor asthma control, such as incorrect inhalation technique, limited knowledge of the disease, poor communication between doctor and patient, and low therapeutic adherence [36]. Factors associated with non-adherence to asthma therapy include difficulties with inhaler devices, complex regimens, side effects and cost of medications [1]. There are also factors unrelated to medications, such as misunderstanding of instructions, fears regarding side effects, dissatisfaction with healthcare professionals, inappropriate expectations and underestimation of severity of diseases. In our study, we found an improvement in adherence over the follow-up period (table 4).

We also observed a similar increase in treatment adherence at visit 2 in each group according to the level of control achieved (not controlled, partially controlled and well controlled), suggesting that in this study poor adherence was not one of the reasons for the limited control seen. However, the percentage of compliant patients detected at visit 2 was surprisingly high in relation to the rates usually found in clinical practice. This high percentage probably reflects the positive short-term effect on compliance of participating in the study, as other authors have suggested [37], and also the tendency of subjective assessments to overestimate treatment compliance. Therefore, the results for adherence should be evaluated with caution.

One possible solution for the poor control related to asthma patients' limited knowledge of the use of antiasthma drugs and devices could lie in the development of education plans with therapeutic guidelines negotiated with the patient in order to encourage adherence [38-41]. We found that patients with "worse knowledge of the treatment", "poor adherence to inhaled treatment" and "uncertainty about the correct use of their inhaled treatment" were the ones with the lowest levels of asthma control.

This is a real-life study that has various methodological limitations, including the absence of a placebo control group and the lack of an assessment of therapeutic adherence or of patients' perception of their disease. An additional limitation was the use of the GINA 2010 strategy, which was valid at the time the study was designed. GINA has recently moved from the concept of "current clinical control" to a two-axes classification for asthma control for clinical management: "symptoms control" and "future risk" including exacerbations, and adverse side-effects of therapy [2]. Compared to GINA 2010, spirometry is recommended in GINA 2014 primarily to assess risk rather than to adjust therapy adjustment. Although this decision was in part based on the high between-visit variability in FEV 1 [2], it was probably influenced as well by the well-established barriers to generalising the use of spirometric studies in clinical practice, such as the cost of the spirometry equipment, the limited time available to perform spirometry and the lack of confidence in measurement and interpretation.

In agreement with other studies, we reported that patients with uncontrolled asthma tended to have more impaired lung function [41, 43]. Lung function assessment can be particularly useful in patients with poor perception of their asthma symptoms, such as elderly patients and patients with associated dysfunctional breathing [41-44].

In conclusion, treatment optimisation as a result of a step increase according to GINA 2010 leads to an improvement in asthma control. We found a lack of agreement between GINA 2010 criteria, including spirometry, and the ACT. We also found that the ACT underestimated asthma control. We observed that treatment adherence and the patients' knowledge of the disease and its treatment are important factors contributing to asthma control. Finally, we showed that age, weight, disease duration, NSAID hypersensitivity and lung function are associated with poorer asthma control in our population.

\section{Acknowledgements}

This study was conducted thanks to the collaboration of the 317 participating investigators.

Authors' contributions: A. Valero, R. Munoz-Caro, A. Torrego, J. Bartra and C. Picado participated in the design of the study, data analysis and writing. R. Palomino performed the statistical design and analysis. All authors read and approved the final manuscript.

\section{References}

1 Global Initiative for Asthma. Global strategy for asthma management and prevention. Revised 2010. www.ginasthma.org Date last accessed: December 1, 2016.

2 Global Initiative for Asthma. Global strategy for asthma management and prevention. Revised 2014. www.ginasthma.org Date last accessed: December 1, 2016. 
3 Gonzalez-Barcala FJ, de la Fuente-Cid R, Alvarez-Gil R, et al. Factors associated with asthma control in Primary Care patients: The CHAS study. Arch Bronconeumol 2010; 46: 358-363.

4 Demoly P, Annunziata K, Gubba E, et al. Repeated cross-sectional survey of patient-reported asthma control in Europe in the past 5 years. Eur Respir Rev 2012; 21: 66-74.

5 Olaguibel JM, Quirce S, Juliá B, et al. Measurement of asthma control according to Global Initiative for Asthma guidelines: a comparison with the asthma control questionnaire. Respir Res 2012; 13: 50-60.

6 Cazzoletti L, Marcon A, Janson C, et al. Asthma control in Europe: a real-world evaluation based on an international population-based study. J Allergy Clin Immunol 2007; 120: 1360-1367.

7 Godard P, Huas D, Sohier B, et al. Asthma control in general practice: a cross-sectional survey of 16.580 patients. Press Med 2005; 34: 1351-1357.

8 Chapman KR, Boulet LP, Rea RM, et al. Suboptimal asthma control: prevalence, detection and consequences in general practice. Eur Respir J 2008; 31: 320-325.

9 Laforest L, Van Ganse E, Devouassoux G, et al. Influence of patients' characteristics and disease management on asthma control. J Allergy Clin Immunol 2006; 117: 1404-1410.

10 Schatz M, Zeiger RS, Vollmer WM, et al. Determinants of future long-term asthma control. J Allergy Clin Immunol 2006; 118: 1048-1053.

11 Partridge MR, van der Molen T, Myrseth SE, et al. Attitudes and actions of asthma patients on regular maintenance therapy: the INSPIRE study. BMC Pulm Med 2006; 6: 13.

12 Nathan RA, Sorkness CA, Kosinski M, et al. Development of the asthma control test: a survey for assessing asthma control. J Allergy Clin Immunol 2004; 113: 59-65.

13 Schatz M, Sorkness CA, Li JT, et al. Asthma Control Test: reliability, validity, and responsiveness in patients not previously followed by asthma specialists. J Allergy Clin Immunol 2006; 117: 549-556.

14 Juniper EF, Bousquet J, Abetz L, et al. Identifying 'well- controlled' and 'not well-controlled' asthma using the Asthma Control Questionnaire. Respir Med 2006; 100: 616-621.

15 Vega JM, Badia X, Badiola C, et al. Validation of the Spanish version of the Asthma Control Test (ACT). J Asthma 2007; 44: 867-872.

16 Vestbo J, Hurd SS, Agustí AG, et al. Global strategy for the diagnosis, management, and prevention of chronic obstructive pulmonary disease: GOLD executive summary (updated 2014). Glob Initiat Chronic Obstr Lung Dis $2014 ; 21-26$

17 Council for the International Organizations of Medical Sciences. International Ethical Guidelines for Epidemiological Studies 1991. http://cioms.ch/publications/guidelines/1991_texts_of_guidelines.htm Date last accessed: to come.

18 Garcia G, Adler M, Humbert M. Difficult asthma. Allergy 2003; 58: 114-121.

19 Bateman ED, Boushey HA, Bousquet J, et al. Can guideline-defined asthma control be achieved? The Gaining Optimal Asthma Control study. Am J Respir Crit Care Med 2004; 170: 836-844.

20 Doz M, Chouaid C, Com-Ruelle L, et al. The association between asthma control, health care costs, and quality of life in France and Spain. BMC Pulm Med 2013; 13: 15.

21 Alvarez-Gutiérrez FJ, Medina-Gallardo JF, Pérez-Navarro P, et al. Comparison of the Asthma Control Test (ACT) with lung function, levels of exhaled nitric oxide and control according to the Global Initiative for Asthma. Arch Bronconeumol 2010; 46: 370-377.

22 Ylldı F; ASIT Study Group. Factors influencing asthma control: results of a real-life prospective observational asthma inhaler treatment (ASIT) study. J Asthma Allergy 2013; 6: 93-101.

23 Terzano C, Cremonesi G, Girbino G, et al. 1-year prospective real life monitoring of asthma control and quality of life in Italy. Respir Res 2012; 13: 112.

24 Díez J de M, Barcina C, Muñoz M, et al. Control of persistent asthma in Spain: associated factors. J Asthma 2008; 45: 740-746.

25 Cazzoletti L, Marcon A, Janson C, et al. Asthma control in Europe: a real-world evaluation based on an international population-based study. J Allergy Clin Immuno 2007; 120: 1360-1367.

26 Peters SP, Ferguson G, Deniz Y, et al. Uncontrolled asthma: a review of the prevalence, disease, burden and options for treatment. Respir Med 2006; 100: 1139-1151.

27 Di Marco F, Verga M, Santus P, et al. Close correlation between anxiety, depression and asthma control. Respir Med 2010; 104: 22-28.

28 Jia CE, Zhang HP, Lv Y, et al. The Asthma Control Test and Asthma Control Questionnaires for assessing asthma control: systematic review and meta-analysis. J Allergy Clin Immunol 2013; 131: 695-703.

29 Khalili B, Boggs PB, Shi R, et al. Discrepancy between clinical asthma control assessment tools and fractional exhaled nitric oxide. Ann Allergy Asthma Immunol 2008; 101: 124-129.

30 Korn S, Both J, Jung M, et al. Prospective evaluation of current asthma control using ACQ and ACT compared with GINA criteria. Ann Allergy Asthma Immunol 2011; 107: 474-479.

31 Thomas M, Kay S, Pike J, et al. The asthma control test (ACT) as a predictor of GINA guideline defined asthma control: analysis of a multinational cross-sectional survey. Prim Care Res J 2009; 18: 41-49.

32 O’Byrne PM, Reddel HK, Eriksson G, et al. Measuring asthma control: a comparison of three classification systems. Eur Respir J 2010; 35: 269-276.

33 Quirce S, Plaza V, Picado C, et al. Prevalence of uncontrolled severe persistent asthma in pneumology and allergy hospital units in Spain. J Investig Allergol Clin Immunol 2011; 21: 466-471.

34 Halbert RJ, Tinkelman DG, Globe DR, et al. Measuring asthma control is the first step to patient management: a literature review. J Asthma 2009; 46: 659-664.

35 Fu J, McDonald VM, Wang G, et al. Asthma control: how it can be best assessed? Curr Opin Pulm Med 2014; 20: 1-7.

36 Kersul A, Balmes S, Rodriguez N, et al. Asthma control. Possible obstacles along the way. Arch Bronconeumol 2010; 46: 8-13.

37 Sumino K, Cabana MD. Medication adherence in asthma patients. Curr Opin Pulm Med 2013; 19: 49-53.

38 Martínez-Moragón E, Serra-Batllés J, De Diego A, et al. Economic cost of treating the patient with asthma in Spain: the AsmaCost study (Study AsmaCost). Arch Bronconeumol 2009; 45: 481-486.

39 Gillissen A. Patient's adherence in asthma. J Physiol Pharmacol 2007; 58: 205-222.

40 Lopez-Vina A. Attitude changes needed to foster treatment adherence in patients with asthma. Arch Bronconeumol 2005; 41: 334-340 
41 Steele AM, Meuret AE, Millard MW, et al. Discrepancies between lung function and asthma control: asthma perception and association with demographics and anxiety. Allergy Asthma Proc 2012; 33: 500-507.

42 Park SY, Yoon S-Y, Shin B, et al. Clinical factors affecting discrepant correlation between Asthma Control Test score and pulmonary function. Allergy Asthma Immunol Res 2015; 7: 83-87.

43 De Groot EP, Duiverman EJ, Brand PL. Dysfunctional breathing in children with asthma: a rare but relevant comorbidity. Eur Respir J 2013; 41: 1068-1073.

44 Cipandri G, Tosca M, Cirillo I, et al. The asthma control in daily practice. Allergy 2016; 71: 907-909. 